\title{
6
}

\section{Neuronal Correlates of Decision Making}

\author{
Michael Platt, Rapporteur \\ Peter Dayan, Stanislas Dehaene, Kevin McCabe, \\ Randolf Menzel, Elizabeth Phelps, Hilke Plassmann, \\ Roger Ratcliff, Michael Shadlen, and Wolf Singer
}

\begin{abstract}
Decision making involves selection from sets of options based on current evidence about the state of the world and estimates of the value or utility of different outcomes. The neural correlates of evidence assessment that guide simple perceptual judgments are now well understood, and we argue that this process can serve as a simple model for organizing behavioral and neural correlates of decision making in more complex contexts, including those that involve economic transactions, social interaction, emotional stimulation, and conscious awareness. Bridging the methodological and theoretical gaps between simple and more complicated decision problems remains a fundamental, but in our view soluble, challenge for understanding the neurobiology of decision making.
\end{abstract}

\section{Introduction}

Over the past ten years, neurobiologists have made significant advances in the understanding of the neural correlates of decision making. Given the nature of this problem, it is perhaps not surprising that the most significant progress has been achieved in understanding the neuronal mechanisms underlying perceptual decision making, in which there is a satisfying match between computational models of behavior and the response properties of single neurons in cortical areas traditionally associated with sensorimotor processing. Building on this work, we have begun to achieve an understanding of the contribution of valuation to this process as well as the neural systems that contribute to learning and applying value to decisions. The extension of this work to social 
interaction, the role of emotions in coloring or modulating the decision process, and the contributions of conscious and nonconscious processes to decision making remain deeply important yet relatively immature fields of inquiry. We predict that important insights can be gained by comparing the principles governing decision processing in diverse animals, like humans and honeybees, which highlight divergent neural architecture. In our report, we outline current understanding of these issues, highlight significant controversies, and pose questions to stimulate new avenues for research.

\section{What Is a Decision?}

Before delving into the neuronal correlates of decision making, let us define what we mean by a decision: A decision is a commitment to a choice or course of action selected from more than one option. We start by considering perceptual decision making, which involves committing to one of a number of propositions about an aspect of the state of the outside world. Such commitment is meant to imply a conversion from an uncommitted state, in which evidence and other factors are considered in relation to all the alternatives, to a state in which one of the alternatives is chosen. Later we will discuss more general decisions, which are typically more divorced from propositions about the state of the world. Viewed as a process, a decision has a beginning and an end, and it evolves over time. Commitment implies termination of the decision process, but it does not imply that a decision cannot be superseded or reversed.

Often, a decision results in an action. If that action is immediate, then the process reveals its time of termination. If there is no explicit action, or if the action is delayed, then there is still a termination, but there is no (obvious) behavioral manifestation. There is good evidence that a single mechanism accounts for termination and choice selection; that is, both when and what is decided (reviewed in Gold and Shadlen 2007). A commitment need not result in an action (immediate or planned) but can instead lead to a choice of strategy or choice to make a new decision about something else or a commitment to implement a complex rule or program (e.g., if told "S1," reply "A1"; else if told "S2", reply "A2"; else wait). It is useful to expand the concept of "choice of action" to incorporate this broader class of decision outcomes.

The key elements of a perceptual decision among $\mathrm{N}$ alternatives include:

1. Identification of alternatives. These are commonly regarded as hypotheses or beliefs about the world. They are ultimately true or false, although this may not be revealed to the decision maker, and are denoted $h_{1}, h_{2}, \ldots$ 
2. Identification of actions: For perceptual decisions, in the general sense developed above, the actions define the states associated with commitment to each of the alternatives: $H_{1}, H_{2}, \ldots{ }^{1}$

3. Evidence is an item of information that has bearing on the alternatives: $P\left(e_{i} \mid h_{j}\right)$. Evidence can originate from a variety of sources and can arrive as a stream in time or all at once.

4. Priors are knowledge about the likelihood of each of the alternatives, absent any evidence; that is, the probability $P\left(h_{i}\right)$ that each of the alternatives is true, before receipt of any evidence.

5. Value and utilities are the consequences of a decision associated with the possible outcomes: $u\left(H_{i}, h_{j}\right)$. Notice that the outcomes include both the actual state of the world and the action of the decision maker. Utilities are based on values and costs, are influenced by motivational and emotional factors, and may be accumulated across sequential choices to define the utility or value of a strategy.

6. Decision variable is a number (or vector of numbers) that combines the preceding terms. An operation on the decision variable establishes the outcome of the decision.

7. Decision rule is often a criterion of the decision variable or a bound to accumulated evidence.

8. Objectives and goals are desired consequences of making decisions. They are usually stated in terms of values, utility, and time.

9. Policy and strategy are operations on control factors (e.g., criteria, decision variable, utility) that influence the decision process to obtain objectives and goals.

Many decisions are not described as choices among discrete alternatives but involve instead an estimate of a quantity or a parameter. For example, instead of deciding whether motion is left, right, up, or down, an observer might be asked to judge its actual direction of movement. It is an open question how and whether the mechanisms of decisions among discrete alternatives can be applied to this problem. In statistical contexts, hypothesis testing and parameter estimation are types of inference. Commitment implies termination of the decision process, but it does not imply that a decision cannot be superseded or reversed.

An alternative perspective that is common in statistical treatments is to separate the hypotheses and actions a little more distinctly. In this case, choices are not treated purely as commitment to hypotheses, but rather as independent

1 It is important to respect the distinction between hypotheses and actions. $P(h)$ refers to the probability that a state of the world holds. It also represents the degree of belief in that state. $P(H)$ refers to the probability that the decision maker will choose a particular outcome. It is not a term that is commonly used, but it could be if the mapping between hypothesis and action were complicated. 
entities in their own right. Further, the state of the world is often considered as being well known, in which case it is only the utilities that are important for determining choices. This encourages a firm functional (though not necessarily an algorithmic or implementational) separation between compiling information about the posterior distribution over the states of world based on the evidence, $P\left(h_{i} \mid e\right)$, and the choice of action as maximizing the expected short- or long-run utility averaging over this posterior. Later, we consider cases in which the state of the world is considered as being well known, where it is only the utilities that are important for determining choices.

\section{Drift-diffusion Models of Perceptual Decision Making}

The neuronal correlates of perceptual decision making have been explored extensively in association cortical areas that intervene between sensory processing and motor affordance (Gold and Shadlen 2007; Sugrue et al. 2004; Smith and Ratcliff 2004; Glimcher 2003; Romo and Salinas 2003). These studies have revealed a number of important features of the decision process. Specifically, the decision process unfolds in time as the output system is continuously fed sensory information that supports a particular motor affordance. For example, the lateral intraparietal area (LIP) contributes to the selection of a target for an orienting movement of the eyes. Neurons in this area continuously query sensory areas for information supporting a particular orienting movement. The value or utility of a particular orienting saccade influences this temporally extended process (Platt and Glimcher 1999; Sugrue et al. 2004).

The temporal unfolding of activity in neurons in the LIP (Shadlen and Newsome 2001; Roitman and Shadlen 2002; Huk and Shadlen 2005) as well as other areas, such as the frontal eye fields (Roesch and Olson 2003; Ding and Hikosaka 2006), the dorsolateral prefrontal cortex (Kim and Shadlen 1999; Heekeren et al. 2004), premotor cortex (Romo et al. 2004), and superior colliculus (Horwitz and Newsome 2001), has been argued to represent a decision variable. Furthermore, the decision variable has been argued to resemble diffusion to boundary models developed to explain choice and reaction time data in psychophysical studies (Ratcliff 1978; Ratcliff et al. 2003). In these models, the decision variable has a starting point, a drift rate or rate of accumulation, and a terminal boundary representing the end of the decision process. According to this view, neurons represent a decision variable that integrates sensory information favoring a particular oculomotor response until the boundary is crossed, after which a movement is initiated. Remarkably, models of this sort can explain a large fraction of the variance in both choice and reaction time based on the spike rates of neurons in many of these areas (Mazurek et al. 2003; Ratcliff et al. 2003).

Complementing the prediction of behavioral data from neuronal activity, computational modeling has also demonstrated that it is possible to fit 
behavioral data with a diffusion class model and predict neuronal firing rates (Ratcliff et al. 2003). Specifically, in a simple brightness discrimination task, a dual diffusion model was fit to behavioral data, accuracy, and correct and error reaction time distributions. Then, position in the process was assumed to represent firing rate, and functions predicting firing rate were able to match most of the main features of the observed firing rate data quantitatively. This correspondence between behavioral models and neurophysiological data are startling, to say the least, given the history of compartmentalization in much of science.

The consilience of the psychophysical model and neurophysiological data thus represents a major success in understanding the neurobiology of decision making. This model has been extended to the neuronal correlates of somatosensory guided decisions (Romo and Salinas 2003), behavioral and imaging data from human subjects performing numerical judgment tasks (Dehaene 2007; Sigman and Dehaene 2005) and object classification tasks (Heekeren et al. 2004). Moreover, similar processes seem likely to underlie decisions about a choice of strategy so the model is not limited to overt actions. For instance, in a dual-task setting, where subjects have to perform quickly, in the order they prefer, a visual numerical judgment and an auditory tone judgment, choosing which of two tasks to perform first adds a stage to the response time which can be modeled as involving a noisy accumulation of evidence up to a threshold (Sigman and Dehaene 2006). It might even be possible to extend these ideas to more complex real-life decisions, as when children select which algorithm (e.g., memory retrieval, explicit calculation, shortcut) to apply to an arithmetic problem, a high-level decision that has been modeled as a differential weighing of evidence for the efficiency of the various alternatives (Siegler 1988).

The model also has the attractive feature that confidence in the decision could, in principle, be estimated from the length of time needed to commit to a response or information encapsulated in integrators that fail to reach the boundary for commitment. We note, however, that thus far computational modeling of psychophysical data does not support the idea that observers use elapsed time as an estimate of uncertainty.

Despite these advances, significant controversies remain. Do all decisions evolve in time? Although it would appear that every decision takes time, it remains unclear as to how the decision process unfolds when there is no relevant sensory information to guide choice. Further, can the model account for decisions extended across long periods of time and involving multiple sources of information, such as choosing whether or not to buy a car? Likewise, decision making at the institutional level may not always involve the extended transmission of evidence. Conversely, legal decision making often involves backward contingency of evidence that alters the decision process.

A second set of questions focuses on whether decisions of this type are more appropriately modeled using nonlinear system dynamics (cf. Wang 2002; Wong and Wang 2006; Machens et al. 2005). These questions notwithstanding, 
the perceptual model of decision making extended in time represents a set of formal, quantitative principles for organizing neurophysiological (and perhaps neuroimaging) evidence gathered in other decision-making contexts, including those that unfold over long periods of time, emotional situations, and social interaction.

\section{The Origins of Variability in Decision Making}

\section{Psychological Models}

One important feature of decision models in psychology is that they assume different sources of noise during processing. There is noise within the decision process, noise in the evidence entering the process from trial to trial, and noise in the decision criteria (or starting point of the process, which is to a large degree equivalent). We would like to emphasize two main points: First, these sources of noise are discriminable in the models. Simulated data can be generated from the model with different values of these noise parameters and these can be recovered. Second, these sources of noise are necessary to fit behavioral data. A diffusion process with equal boundaries predicts that reaction times on correct and error trials are identical. For example, variability in evidence (drift rate) from trial to trial leads to errors slower than correct responses and variability in decision criteria from trial to trial gives fast errors.

\section{Noise and Randomness in Decision Making}

Most decisions of interest from a psychological, economic, or evolutionary point of view appear stochastic. In part, this is because evidence and/or valuation does not uniquely guarantee only one optimal choice. Moreover, the neuronal correlates of the assumed decision variable can only be revealed when there is incomplete agreement between information and choice. For these reasons, psychological and neurobiological research has focused on decisions based on evidence and/or valuation that tends to favor, but does not determine, one choice. In these situations, knowledge of the variables influencing choice appears to govern the probability of an outcome without guaranteeing it. Thus, the actual choice appears to be random.

There are two qualitatively different classes of mechanisms which could give rise to probabilistic behavior (Shadlen et al., this volume; Luce and Suppes 1965). The difference between these two mechanisms is whether the noise is in the assessment of evidence and utilities (first stage) or in the application of the decision rule (second stage). If it is in the first stage, then the decision arises deterministically based on the sign of a noisy decision variable relative to a criterion. In this case, variability arises because the representation of evidence is intrinsically noisy (Shadlen and Newsome 1994, 1998). This model is 
similar to the random utility model of Manski (1977), which assumes that the decision rule is applied deterministically. This model implies that individual preferences or utilities will not always be the same under identical conditions because of measurement error and random variation in the assessment of preference or utility. Utilities are thus conceptualized as random variables.

If the noise is in the second stage, the decision process uses errorless representations of the utility of options to compute a probability of choice and realizes it in an explicitly random act, equivalent to flipping a weighted coin (Glimcher 2005). This model is similar to the constant utility theory of Luce (1959), which states that choice is a probabilistic function of preferences or utilities (Luce 1959). Thus, accordingly, utilities can be deduced from the relative frequency with which an individual chooses various alternatives under identical conditions.

These two models of probabilistic behavior cannot be distinguished behaviorally. An animal that chooses "right" on $71 \%$ of trials could be choosing "right" every time the value of a normally distributed variable with $\mu=\sigma=1$ exceeds 0 , or it could be doing so by flipping a weighted coin on each trial $(p=0.71)$. For well-studied perceptual decisions, the first idea has strong support (see Gold and Shadlen 2007). Enthusiasm for the deliberate random act derives mainly from theoretical claims that randomness confers survival advantage in predator-prey situations and competitive games (Glimcher 2005; Maynard Smith 1982).

\section{Neuronal Noise and Decision Making}

In perceptual decisions, variability in choice and response time can be traced to the variable activity of neurons in the cortex (for a review, see Parker and Newsome 1998; Mazurek and Shadlen 2003). Recordings from the neurons that represent evidence and the neurons that represent the decision variable show trial-to-trial variation in spike rates which explains (a) the behavioral sensitivity overall, (b) errors on single trials, and (c) some of the variance in reaction time. This implies strongly that although, in principle, behavioral variability could have a variety of causes - including the representation of states that are simply unknown to the experimenter or irrelevant to the task at hand - the brain is unable to eliminate these causes. It is affected by variability in the way that conforms to the noise in the engineering sense, and this leads to mistakes.

What is the source of the neural noise? This is an immense topic beyond the scope of our report. We note, however, that there is clear and compelling empirical evidence that cortical neurons discharge irregularly (Shadlen and Newsome 1994, 1998). This implies that every quantity that the neocortex represents - be it evidence, a decision variable, or a representation of elapsed time-is corrupted in some sense by response variability, and thus includes both signal and noise. Although noise may be an intrinsic feature of the biophysical properties and synaptic connections of cortical neurons, this does not 
exclude the possibility that intrinsic neuronal noise has been exploited by evolution to promote behavioral variability or flexibility, which can have obvious survival advantages (Glimcher 2005).

\section{Value-based Decision Making}

As noted, decisions involve selecting among possibilities based partly on their value or utility. We can define the value of a state as the expected long-run summed reward, discounted by the delay to its receipt, starting from that state; the value of an action at a state (sometimes called a $\mathcal{Q}$ value) can be defined as the expected long-run summed reward starting from initiating that particular action when in that state, again discounted by delay. From a formal perspective, it is appropriate to consider net rewards after costs are taken into account, although the neural realization of costs and punishments is less clear than that for rewards.

As mentioned, much of the work on perceptual decision making has focused on uncertainty about the state of the world and the decision variables that accumulate sensory information favoring one state over another. In contrast, the tradition of studies investigating complex, long-run values has tended to assume that states are well known, thus placing the emphasis squarely on the utilities. This tradition is the focus of our discussion for the remainder of this section. However, two important caveats point to the future merging of these approaches: First, although the state of the subject in the world may be known, the rules of the world (e.g., what transitions between states are occasioned by which actions) are not. In this case, subjects face an exploration-exploitation dilemma associated with their uncertainty about the nature of the environment. Although not generally couched in such terms, this implies that there exist some higher-order decision variables that control the course of exploration. Second, value-based decision making still requires a physical realization of competition among the options. This likely involves the same sort of mechanisms as discussed for perceptual decision making, and it may be possible to endow the variables coded by neural firing rates in the competing populations with some form of decision variable semantics.

\section{Value Systems}

Consideration of the psychological and neural data suggests that value thus defined is not a unitary concept: there are different ways of defining and calculating value, and there are different ways of using values to control decisions. These different notions of value also appear to map onto somewhat distinct neural systems. Available data suggest the existence of four different systems for computing and representing value, although there is by no means consensus that this is the only scheme that can adequately encapsulate all available data: 
1. The first value system is the native value system. Native values are influenced by motivational states (e.g., hunger, thirst) and (probably) mood. Thus, native values may differ from true, real-world values. This system has been described as the "liking" system (Berridge and Robinson 2003) and is most likely mediated by the primary taste system, hypothalamus, and periaqueductal gray region of the brainstem and can be modulated by opioids and benzodiazepines.

2. The second value system is the forward model system. This system learns a full tree of states, actions, transitions, and outcomes, and it infers values by extensive search of this tree. Since the forward model's evaluation of the outcomes it encounters during this search appears to be sensitive to motivational state, it is goal directed. This model of value is computationally hard to compute and is probably the best candidate to be considered as an explicit value system. The neural systems associated with outcome evaluation in the forward model remain a subject of debate, but may be related to physiological evaluation mechanisms (discussed further below). The inference process appears to involve the dorsolateral prefrontal cortex as well as the dorsomedial striatum and places a heavy burden on working memory.

3. The cached value system represents the learned value of a state or an action, which is typically independent of motivational state or changes in the environment. Thus, the cached value system is inflexible and cannot generalize to different motivational states or contexts. The normal form of learning uses dopamine to report an appetitive prediction error, with values being represented in the amygdala and possibly the ventral striatum. It has been speculated that serotonin calculates cached values for aversive outcomes (Daw et al. 2002).

4. The fourth value system computes the long-run expected value, which putatively acts as an opportunity cost of inaction and controls the vigor of responding. This value system is important for determining when to act and may be linked to tonic dopamine levels (Niv et al. 2006).

\section{Value-based Control Systems}

These four value systems contribute in a variety of ways to four different systems controlling decision making:

1. Goal-directed control follows from state-action values computed by the forward model. Since choices are based on extensive search of all states, actions, and outcomes, goal-directed decision making provides a sophisticated, but computationally expensive, control system.

2. Habitual control operates on cached state-action values. Because cached values are insensitive to motivational state, habit-based control is inflexible. 
Habitual control depends strongly on the dorsolateral striatum and is influenced by the neuromodulators, dopamine, and serotonin.

These two control systems are conventionally assumed to compete through a softmax function (essentially rolling dice) according to their relative uncertainties, although the mechanisms that compute uncertainty remain unclear. Competition within each system could possibly be implemented instead by a diffusion-to-bound mechanism, similar to the one proposed to account for perceptual decision making (Shadlen et al., this volume; Gold and Shadlen 2007). The long-run expected value influences action latency.

3. The episodic controller can best be viewed as a replacement for the forward model because it is more accurate in very early learning. This system simply recommends repeating any action or sequence of actions that was successful in the past, a strategy that can be shown to be optimal (i.e., to have minimal uncertainty) in some particular circumstances. The hippocampus likely plays a key role in episodic control.

4. The Pavlovian controller specifies actions regardless of their instrumental suitability. This control system consists of intrinsic, instinctive mechanisms that have been programmed by evolution due to their routine efficacy. Pavlovian control includes spontaneous approach and avoidance behaviors in the light of value-based predictions of reward, and thus leads to behavioral phenomena such as autoshaping, negative automaintenance, and Pavlovian-instrumental transfer. Preparatory appetitive and aversive effects appear to depend on the ventral striatum, whereas more consummatory defensive and aggressive Pavlovian responses appear to depend on the dorsal periaqueductal gray region of the brainstem.

These four value systems and associated decision control systems can account for a wide array of observations following specific manipulations (e.g., such as incentive devaluation) as well as damage to specific brain areas (e.g., the dorsolateral striatum), and these promote particular behavioral responses. There are, however, other conceptualizations of value and control systems that might equally account for these observations. This caveat becomes clear when we consider precisely how a single decision evolves in time within and across these systems. Specifically, how can evidence for these four systems be reconciled with neurophysiological observations of integration with respect to time in perceptual decision making? Do the resultant values computed in each of these four systems contribute to the decision variable apparently computed in cortical areas mediating sensorimotor processing? If so, how do they influence the decision variables? It is not yet clear precisely how competition between decisions is instantiated (i.e., whether decision variables are parsimoniously seen as being involved) and what influence uncertainty has on states (as in perceptual decision making). For instance, there is evidence for value-related bias of the decision variable (Platt and Glimcher 1999; Sugrue et al. 2004; 
Klein et al., in prep.) as well as motivationally induced changes in the rate of integration (Bendiksby and Platt 2006) of neurons in the LIP. Certainly, the mechanisms underlying value-related influences on the decision variable, as well as precisely where value-related signals enter the decision process, remain to be explicated fully, thus providing a fruitful avenue for future research.

\section{The Role of Emotion in Decision Making}

\section{Value and Emotion}

As discussed above, the concept of value is fundamental to current models of decision making. When considering the impact of emotion on decision making, one might begin by considering the relation between value and emotion.

Current models do not limit emotion to a subjective feeling. There is no question that many of us make choices when we do not have strong feelings. One does not need to have a strong subjective experience to invoke an emotional process (LeDoux 1996). The distinction between feeling and emotion is prominent in current models of emotion, but it is less familiar to those from other disciplines and can thus result in some confusion.

What distinguishes value from emotion? The answer to this depends on how we understand the purpose and limits of emotion. It is has been argued by some that emotion is a relevance detector in that it allows us to determine what is or is not important in a given situation, in order to generate an adaptive response (Cosmides and Tooby 2000). In this broad framework, we can clearly see the intersection of emotion and value. Although emotion may encompass more than value, value is a fundamental component of emotion. However, others have argued that systems that mediate emotion as well as those that do not both contribute to the calculation of value (Cohen 2005). Accordingly, emotion and value are clearly related, but remain distinct. This latter view is more prominent in current models of decision making.

\section{What Is Emotion?}

Prior to considering the role of emotion in decision making, it is necessary to define precisely what we mean by the term "emotion." Emotion is often discussed as a unitary concept in the decision-making literature, but there are clearly a number of components that should be differentiated if we are to understand the range of means by which it might impact our choices. How to best characterize the components or dimensions of emotion has been widely debated by psychologists and philosophers, and there is no clear agreement. As a working framework, we will refer to Scherer's (2000) model of emotion.

In Scherer's model, emotion refers to synchronized responses, which may include physiological activation, motor expression, subjective feeling, and 
action tendencies, that are triggered by internal or external events and which are of significance to the organism. Mood reflects a more diffuse, long-lasting, and likely less intense state and is characterized primarily by a change in subjective feeling. Attitudes are relatively enduring, affectively colored beliefs, preferences, and predispositions toward objects or persons. Personality traits are emotionally laden, stable dispositions, and behavioral tendencies typical for a person.

These four distinctions represent classes of states one might include under the term "emotion." Within each class, one might further differentiate specific types of responses or states, such as fear, happiness, sadness, or social emotions (e.g., guilt). Another common distinction in emotion research is to characterize reactions or states with dimensions, such as valence (i.e., positive or negative) and arousal (intensity). As this framework makes clear, the term "emotion" is often used to represent a class of processes, each of which might have a unique impact on decision making.

\section{Current Models of Emotion and Decision Making}

\section{Dual Selves Models}

One of the main ways that the impact of emotion on decision making has been characterized is to view emotion and cognition as two alternative means by which we can arrive at a choice. The notion that we can make choices with either our heart (emotion) or our head (reason) was first proposed in early philosophical writings, and this distinction persists to this day in common language use, legal reasoning, as well as scientific investigations. A primary contention of this dual selves approach is that emotion and reason compete with one another when arriving at a decision.

As the popularity of the dual selves model suggests, there is clearly some intuitive appeal in viewing decision making this way. However, there are also numerous problems which lead us to suggest that the model is simply too simplistic, as well as unrealistic, to capture the role of emotion in decision making.

For example, one function of emotion is to allow us to interact adaptively with our environment, thus avoiding situations that are dangerous or threatening and moving us toward choices that are likely to increase evolutionary fitness. Emotion plays an important role in the assignment of value. In this way, emotion is a critical component of models of decision making.

Another primary difficulty with the dual selves model is that it suggests a unitary role for emotion in decision making. As discussed below, there are several means by which different components of emotion can influence decisions, and no single characterization will suffice. Although one might argue that under some circumstances an emotional reaction might interfere with one's ability to reason about a decision, there are also several other ways that emotion might influence decisions. How cues from emotional reactions and states 
are integrated into the "rational" decision-making process has yet to be fully understood. There is, however, abundant evidence to suggest that the relation between emotion and reason in decision making is not simply competitive.

\section{Somatic Marker Hypothesis}

One model for emotion's role in decision making that has received considerable attention is the somatic marker hypothesis (Damasio 1996). Damasio and his collaborator Antoine Bechara suggest that emotion can provide a heuristic for decision making by associating physiological bodily reactions, called somatic markers, with specific choices or options (Bechara and Damasio 2005). When faced with a decision, the somatic marker allows one to assess the potential emotional consequences of a possible option without having to use reason. In this way, emotion provides a shortcut for fast, intuitive decisions and does not depend on deliberative assessment or awareness of potential outcomes. It is suggested that the ventral medial prefrontal cortex and the insular cortex play critical roles in representing somatic markers.

Although it is likely that physiological emotional reactions - and their learned associations with objects, persons, or potential outcomes - play an important role in decision making, there are several aspects of the somatic marker hypothesis that have not been supported by the data.

One problem relates to how the physiological response is mapped onto a particular choice. In the initial Bechara gambling task (Bechara et al. 1994), it was proposed that when considering a possible option, an increased physiological response (i.e., somatic marker) causes one to move away from that option (which is riskier and, ultimately, less rewarding) toward another that does not invoke as strong an emotional response. However, simply changing the order or outcomes of the possible options has been shown to lead to the opposite result; that is, a stronger physiological response for the preferred option (Tomb et al. 2002). In other words, somatic markers do not predict the appropriate action.

In addition, a dissociation between the physiological response and choice has been demonstrated in a number of studies. Patients who do not have a normal representation of bodily states perform normally on the Iowa gambling task used in the initial Bechara studies (Bechara et al. 1994). In addition, lesion evidence in rats suggests that the neural circuits mediating the physiological response to a conditioned fear stimulus are partially distinct from those mediating the decision to avoid the conditioned stimulus (Amorapanth et al. 2000).

\section{Components of Emotion and Decisions}

In spite of difficulties with some of the strong claims of the somatic marker hypothesis, one of the advances of this approach is that it considers the independent contribution of a specific component of emotion: the physiological 
response. This component approach has been extended in recent work by Lerner and colleagues (Lerner and Keltner 2001).

There is evidence, for example, that distinct emotional reactions, each of which leads to a similar physiological response and bodily state, can have the opposite effects on decision making. Despite years of investigation, researchers have been unable to detect clear differences in the physiological responses that characterize discrete emotional states, such as anger and fear. For this reason, and others, emotion researchers have suggested that an appraisal of the situation is an important component of the subjective qualities of an emotional response. Lerner and Keltner (2001) suggest that anger and fear, which elicit a similar physiological profile, can have opposite effects on the assessment of risk in decision making. Anger, along with happiness, results in less risk aversion, whereas fear leads to increased risk aversion. This effect was observed both when assessing anger and fear as personality traits and when experimentally manipulating mood. Lerner and Keltner (2001) suggest that this reflects differences in the cognitive sense of certainty and control between anger and fear. An alternative interpretation is that anger and fear promote different action tendencies (i.e., approach and avoid). In either case, it is clear that the physiological state alone cannot determine emotion's influence on the assessment of risk. In a related series of studies, Lerner and Lowenstein (2003) demonstrated that the induction of a sad or disgusted mood differentially influences the endowment effect, with a sad mood essentially reversing the endowment effect and a disgusted mood simply eliminating it.

\section{Proposed Framework for Future Investigations of Emotion and Decision Making}

As the studies by Lerner and colleagues cited above demonstrate, examination of the components of emotion reveals a range of effects on decision making, including opposite patterns depending on the specific emotional state. To date, much of the research on emotion and decision making has been rather imprecise in defining the specific aspects of emotion that are important. Given the range of processes and functions encompassed by the term emotion, future research on the neural mechanisms of emotion and decision making would benefit from a more precise characterization and assessment of emotion and its impact on specific aspects of decision making.

In addition, it is clear that decision making can also impact emotion. For instance, one can choose to engage in a cognitive strategy to regulate one's emotional state (i.e., the glass is half full). This choice may not only influence emotional reactions, but also subsequent decisions made while in this state. In addition, choosing an option can represent an investment in that option, which in turn can change its perceived value. Through processes such as cognitive dissonance, social psychologists have identified a range of means by which our choices will impact our attitudes and subsequent decisions. Clarifying the 
complex interactions of emotion and decision making, and the relation to value, is an important challenge for the future.

Finally, we suggest that an initial approach to understanding the mechanisms of emotion and decision making might be to link specific components of emotion to aspects of existing models of decision making. For instance, we suggest that specific emotional states and reactions elicit action tendencies, such as fear and withdrawal or anger and approach. In the context of the particular model of value-based decision making discussed above, these action tendencies are likely reflected in the motivational state in the native value system and forward model system (Dayan, this volume). Similarly, one might incorporate the impact of physiological arousal in the assessment of outcomes in this model or take advantage of a detailed description of emotion to further characterize components of the value-based model, such as behavioral vigor or the Pavlovian controller.

We have provided examples from only one model, but it is the approach that we wish to emphasize here. Although there are components of the mechanisms of emotion that are clearly distinct from those of decision making, there are likely several levels of integration. By emphasizing the integration of models of emotion and decision making in future research, we can avoid the implications of the intuitively appealing, but inaccurate, dual selves models which have dominated both the layperson's perceptions and research on emotion and decision making.

\section{Conscious and Subliminal Decision Making}

\section{Defining and Measuring Conscious and Unconscious Processing}

Descriptions of valuation and decision-making systems at the functional and neurophysiological levels often remain agnostic about whether these processes occur at a conscious or nonconscious level. To address this, we must find minimally different conditions in which availability of conscious information varies, measure the extent to which information is processed consciously and nonconsciously, and provide a means for measuring the influence of these processes on valuation and decision making (Dehaene, this volume).

There are, in fact, many ways to study the conscious/nonconscious contrast (e.g., blindsight, forgetting, hemi-neglect, masking, attentional blink). Typically, one compares two conditions that are as minimally different as possible, yet promote either conscious or nonconscious processing; for instance, a word followed by a mask (Kouider and Dehaene 2007). Asserting whether processing is or is not conscious, however, can be difficult. The key feature of conscious processing is the subjective awareness of an event. This is usually assessed by reportability; that is, the ability to give a verbal or nonverbal report or 
"commentary" on the event. However, when a subject is unable to report, there is no universal agreement that this indicates a lack of subjective awareness.

Nonetheless, reportability seems to be an excellent index of a major transition occurring in the availability of information for many tasks. For example, masking tasks require subjects to perform a discrimination as well as provide a verbal report about a stimulus followed by a mask. The delays between stimulus and mask determine reportability as well as discrimination performance on this task. Although subjects often perform better than chance when the stimulus remains subliminal, objective performance measures and subjective reportability share a similar threshold: a value of the delay beyond which stimulus information is available for discrimination, report, or, in fact, essentially any task (Del Cul et al. 2007).

\section{Influence of Conscious and Subliminal Processing on Decision Making}

Neurobiological evidence suggests that nonconscious events can influence the decision processes that integrate evidence favoring a particular motor response, as outlined above. For example, subliminal priming can evoke biased activation in motor structures associated with the behavioral report (Dehaene et al. 1998). Furthermore, changes in reaction time associated with subliminal processing can be explained by partial accumulation of evidence provided by the prime (e.g., Vorberg et al. 2003). These observations suggest that the temporal accumulation of sensory evidence can operate without conscious processing. Importantly, this can occur at a fairly high level of processing since subliminal primes can be processed in terms of their semantic content (Dehaene et al. 1998) or motivational value (Pessiglione et al. 2007). Recently, subliminal cues have even been found to influence executive-level processing such as task switching (Lau and Passingham 2007).

Despite the importance of nonconscious processing for decision making, items or events that enter awareness can differentially influence the decision process. Numerous differences between subliminal and conscious-level processing have been proposed (Dehaene and Naccache 2001). Subliminal primes typically have only a minimal subthreshold influence on behavior, altering task-related choices by a few tens of milliseconds and error rates by a few percent. This is compatible with the hypothesis that many if not all of the brain's evidence accumulation systems can begin to operate nonconsciously; however, it also suggests that crossing the threshold for a full-blown decision is often, if not always, associated with conscious-level processing. Usually, subliminal primes have a short-lived influence on decision processes that does not continue beyond a few hundreds of milliseconds; this suggests that active maintenance of information is somehow tightly correlated with conscious-level processing. Finally, there is evidence that some inferential and/or executive processes depend on conscious-level reflection. For example, if in a conflict task the prime conflicts with the target on a majority of trials, such that subjects 
can exploit prime information to anticipate an opposite target, this information can only influence the decision process when the prime is consciously perceived (Merikle and Joordens 1997). Conscious-level processing seems needed when the decision involves non-automatized changes in high-level processes; for instance, the strategic changes that adapt decision parameters such as the decision threshold from one trial to the next as a function of perceived task difficulty (the Gratton effect; see Kunde 2003).

\section{Neural Systems Supporting Conscious and Nonconscious Processing}

These observations raise the question of the neurobiological substrate of conscious and nonconscious processing. There is some evidence that conscious processing involves large-scale interactions between diverse brain areas, which may be evident in synchronized electrophysiological responses detectable on the scalp using EEG. Experiments exploiting the phenomenon of binocular rivalry suggest that access to conscious processing is associated with enhanced synchronization of neuronal responses (Fries et al. 1997, 2002; Tononi et al. 1998). This notion has received further support by the demonstration that the earliest electrographic correlate distinguishing conscious from nonconscious processing is a transient increase of precise phase synchronization of oscillatory activity in the gamma frequency range across a widely distributed network of cortical areas. This transient phase locking occurs around $180 \mathrm{~ms}$ after stimulus presentation and appears to be the trigger event gating the access of signals to the work space of consciousness (Singer 2007; Melloni et al. 2007). The subsequent transfer and maintenance of consciously processed information to working memory is accompanied by sustained oscillatory activity in the theta frequency range, again involving a widespread network of cortical areas, and by enhanced gamma oscillations during recall (Melloni et al. 2007). Dorsolateral prefrontal cortex is thought by many to be a key node for conscious processing and is frequently activated or found synchronized with posterior areas in fMRI contrasts of conscious/nonconscious processing. Intriguingly, electrophysiological markers for conscious processing take approximately 200 $\mathrm{ms}$ to develop and are therefore not evident in ERP components, such as the N170 (Del Cul et al. 2007; Sergent et al. 2005), but consciously processed stimuli lead to an enhanced P300 component which is considered as a correlate of the transfer of information to working memory (Melloni et al. 2007). Later, ERP components can be directly related to reportability using temporal accumulation models. Subliminal stimuli may fail to create enough cascading activation to activate large-scale circuits in an effective, coherent manner, and thus their effects on the decision process may be weaker and restricted to a small set of task-related processes.

An important open issue concerns the relation of these observations to top-down attentional control, which evokes similar patterns of neural activity. Attention and conscious processing are expected to share some features, 
since awareness requires selection of some events or stimuli over others. In this context, it is noteworthy that focused attention does not only lead to enhanced responses to the selected stimuli but also to an anticipatory entrainment of neuronal populations into coherent gamma oscillations (Fries et al. 2001). Despite this covariance, the neural mechanisms underlying attention and consciousness are separable.

\section{Outstanding Questions Regarding Conscious and Nonconscious Processing}

One question that we pondered was whether consciousness as a process might be construed or modeled as a nonconscious decision process to engage or report (Shadlen and Kiani 2007). Such an analogy might help to explain the apparent consistency between discrimination performance and reportability on a trial by trial basis observed in human subjects. This observation suggests that the representation of evidence and its accumulation are intact, whereas the policy that guides and implements decision termination is faulty. If conscious processing reflects an integration process for access to report, then one would predict that reportability and discrimination performance curves should be related by a criterion shift. This model could thus help to provide a description of a mechanism that can be linked to the neural correlates of conscious processing. Additional ideas suggest the importance of working memory in conscious processing. For example, working memory storage is more efficient for consciously perceived stimuli than those that remain subliminal.

One important question is to what degree such conscious processing, and its influence on decision making, is confined to humans. Addressing this question requires developing behavioral techniques that encourage subjective reportability in animals. This has been achieved in studies of binocular rivalry (Logothetis and Schall 1989) as well as blindsight (Cowey and Stoerig 1991). Such evidence supports the notion that some animals may process information both consciously and nonconsciously, but it remains to be determined whether there are quantitative differences between species or whether these differences might be qualitative.

Another relevant question relates to how we can provide a neurobiological understanding of the qualia of consciousness. There may be signatures in behavior, such as confidence ratings, that are measures of qualia. Formal models may permit us to determine whether qualia and reportability represent distinct aspects of the mechanisms associated with conscious processing.

A final question relates to the interfering effects of consciousness on decision making. In general, nonconscious processing is inefficient; in subliminal priming, for example, only a small subthreshold amount of information is available to bias decisions. However, certain tasks, such as swinging a baseball bat or searching for the name of the author of a paper from 1967, may be better performed without conscious awareness. Indeed, we may consciously banish 
such tasks to unconscious processing to solve them more efficiently. Understanding such nonconscious incubation phenomena and how they eventually lead to "aha" moments seems particularly important for understanding creativity and insight. When people engage in explicit, verbal reflection, this interferes with finding a solution by insight but does not interfere with solving logical problems using analytic techniques. Moreover, explicit, deliberative processing seems particularly disruptive for aesthetic judgments. Such observations suggest that conscious and nonconscious processes can be complementary and must be actively integrated, yet how these processes differ (what are the powers and limits of nonconscious processing) and what the neural mechanisms underlying these processes are remain to be explored. Further, just how these processes are related to habitual systems underlying valuation and decision making remains to be determined.

\section{Accounting for Individual Differences in Decision Making: Sources of Heterogeneity in the Decision Process}

An important, but unresolved, question is how we can account for differences in decision making between individuals, age groups, neurological conditions, and behavioral contexts. Perceptual and economics-based models provide two different but complementary approaches to address this issue.

\section{Perceptual and Cognitive Decision Making}

At the simplest level, differences in perceptual decision making can be usefully studied using the diffusion-to-bound model described above. This approach has been applied to individual and group differences in a range of experimental tasks, including numerosity discrimination, letter discrimination, brightness discrimination, recognition memory, and lexical decision. In all tasks except letter discrimination, the evidence extracted from stimuli is the same when performed by college students or 60-75 year olds (Ratcliff et al. 2006). In contrast, letter discrimination with masking shows a decrease in evidence accumulation with age because older adults progressively lose higher spatial frequency vision. There is a modest slowing of nondecision components with aging, but the main source of longer reaction times is the application of a more conservative decision criterion. In some discrimination tasks, model parameters change systematically with age during childhood (i.e., nondecision components speed up and decision criteria are reduced), whereas for other tasks there is a dramatic increase in drift rates by age 9 or 10. There is a range of drift-diffusion model parameters expressed in the neurologically intact young adult population.

This style of analysis can also be applied to behavioral changes associated with neurological disorders. For example, in lexical decision tasks, patients with aphasia show drift rates in the normal range, but their decision criteria 
is impaired relative to neurologically intact individuals. This suggests that the representation of evidence and its accumulation are intact, whereas the policy that guides and implements decision termination is faulty. Because similar models have been successfully applied to studies of the neuronal correlates of decision making, this approach shows great promise of directly linking individual differences in decision components to the underlying neurobiology.

\section{Social and Economic Decision Making}

Traditionally, economists have explained heterogeneity in observed behavior either through differences in preferences, differences in information sets, or the selection of different equilibrium strategies. Neuroscience can help document and further explain the sources of these differences, for example, by revealing the variances in information-processing strategies, computational capacity, or valuation systems. This will be enhanced by directly linking these variances to quantifiable components of the decision architecture revealed in neurophysiological studies.

One possible explanation for variance may be due to the heterogeneity of preferences or valuations in the brain. For example, in the gambling task depicted in Figure 6.1, person 1 must choose $a$ or $b$, against Nature, $N$. In standard theory, we can talk about $U(10), U(15)$, and $U(0)$ as the subjective utility of the monetary outcomes 0,10 , and 15 . Assume Nature is governed by the following random process: play left with probability $p$ and play right with probability $1-p$. This can then be solved using an expected utility calculation:

$$
U(10)=p^{*} U(15)+\left(1-p^{*}\right) U(0),
$$

where $U^{\prime}>0, U^{\prime \prime}<0$, and $p^{*}$ is chosen to give the equality. One reason people may show different patterns of choice in this situation (i.e., have different $p^{*}$ ) is because their preferences, $U$, may be different. Supporting this notion, parametric brain activation correlates of different preferences have been reported in the parietal cortex of people playing a gambling task (Huettel et al. 2006). Although the reasons why people might hold different utility function remains to be determined fully, it seems likely that genetic background, developmental trajectory, and context reactivity contribute to differences in the neural architecture that supports valuation, emotional responses, evidence accumulation, and the decision threshold setting mechanisms outlined above.

A second reason people make different decisions may be because they process information differently. In the voluntary trust game (Figure 6.2) by McCabe and Smith (2000), player 1 moves left $50 \%$ of the time. In response, player 2 moves right (reciprocated) $75 \%$ of the time in one-shot play. This occurs more frequently than predicted by the Nash equilibrium of player 1 always moving left and player 2 always moving right. For those players moving left, their estimated $q$ must have been less than $q^{*}$, and for those moving right, their estimated $q$ must have been greater than $q^{*}$. 


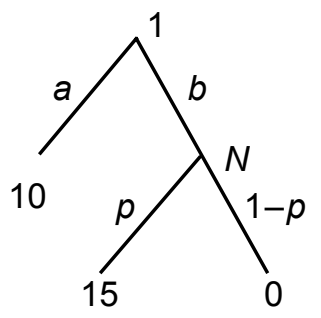

Figure 6.1 Gambling task: Choose $a$ or $b$ against Nature $(N)$.

It is difficult to imagine that this strong reciprocity is simply the result of differences in risk aversion, but instead seems more likely to be due to social factors. For example, player 1 (who played right) may have thought that this would create a social obligation for player 2 to move left-a notion supported by behavioral observations in the involuntary trust game (Figure 6.3; McCabe et al. 2001). When player 1 is forced to move right and player 2 could not see player 1's foregone opportunity cost, player 2 was twice as likely to play right when compared to player 2's behavior in the voluntary trust game. Furthermore, McCabe et al. (2001) found that cooperators showed significantly greater activation in the anterior paracingulate cortex when playing other humans than when playing against a computer. This area has been found to be active in numerous fMRI experiments examining theory-of-mind (Gallagher and Frith 2003), suggesting social contexts and interactions promote approach behavior, engage reward systems in the brain, and encourage empathy and social reasoning.

A third reason people vary in their decisions could be due to the use of different strategies in playing the game. Krueger et al. (in preparation) look at repeat play of the voluntary trust game played by the same partners who alternated roles between player 1 and player 2. Consistent with earlier behavioral findings, they find that players can be divided into two types: unconditional and conditional cooperators. Intriguingly, these two types of cooperators exhibit different neural signatures in the brain. Thus, individual differences in decision making may arise from differences in strategy identified with distinct patterns of neural activity.

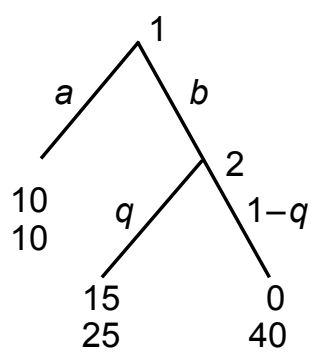

Figure 6.2 Trust game: Choose a or $\mathrm{b}$ against person 2 . 


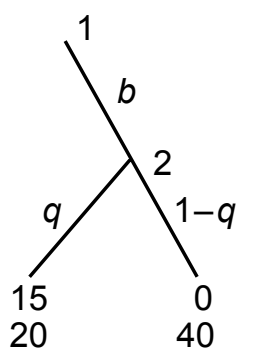

Figure 6.3 Involuntary trust game: Choose $a$ or $b$ against person 2 .

The economic approach to understanding the heterogeneity of decisions made by different people or promoted by different contexts provides a tantalizing hint at the complexity underlying real-world social decisions. Despite the attractiveness of this approach, however, considerable questions remain unanswered. This reflects, in part, the relative maturity of neuroeconomics relative to the neurobiology of perceptual decision making. Importantly, although it is relatively easy to describe the equilibrium point in a game formally, it seems much harder to map this number onto underlying neurobiology. What would an equilibrium look like at the neurophysiological level? In social situations, there is an explosion of degrees of freedom that all must, ultimately, be reduced to a single quantity, the decision variable, in order to inform choice. How could an equilibrium be instantiated in a decision variable that is integrated over time to a threshold for commitment?

\section{Extending Neuronal Models of Decision Making to Long Temporal Scales}

It seems unlikely that simple accumulation models will be able to be extended to long timescales, such as decisions over hours or days. Over intermediate timescales, a model such as Busemeyer's decision field theory may apply (Busemeyer and Townsend 1993, 1995). For example, in buying a car, one may pay attention to different features of the stimulus (e.g., gas mileage, color, power). Decision field theory assumes that these features drive a single decision process - at each time step, one of the features drives the process towards one or the other possible options. Over tens of seconds or a few minutes, this seems a reasonable candidate that connects well to established models of perceptual decision making and to our current understanding of the neurophysiology in which the decision process is embodied.

However, in tasks that require minutes, hours, or days, (e.g., planning out a chess move, deciding whether to go to a conference, and so on), a decision will almost certainly involve multiple steps which take some information computed as intermediate products that are required by later stages for the final decision 
or choice. In addition, intermediate results will have to be stored and retrieved when the decision process is interrupted, for example, by boisterous children, work, or the television. Because the timescale can be long, behavior will depend on the combinations of the various stages of processing and not so much on the individual steps. Of course, in the range of a few seconds, behavior will be determined by the distribution of the individual decisions; however, when decision times are tens of seconds or longer, the organization of stages will then dominate. Connecting long timescale decision processes to the underlying neurobiology will require both reasoned computational modeling and improved understanding of the neural processes that permit information to be accumulated, stored, evaluated, and connected over long delays.

\section{Evolution of Neural Architectures for Decision Making}

As large-brained primates we often neglect the fact that quite complex decision making can be accomplished by much smaller and differently organized nervous systems. There is an enormous richness and complexity of behavior that appears to occur without any conscious processing. In fact, one might argue that such behaviors are better than conscious; that is, they are highly efficient, infallible, fast, and exquisitely adapted to a local environment. Although many of these behaviors reflect the ineluctable releasing of a motor pattern by a sign stimulus, many more behaviors appear to rely on flexible processes quite similar to those guiding behavior in humans and other vertebrates. These observations raise the important question of whether similar computational rules underlie the decision process when it is embodied in dramatically different nervous systems.

This complexity is demonstrated aptly by the behavior of the honeybee (Menzel et al. 2007). These diminutive volant insects use the well-known waggle dance to communicate navigational information to others in the hive. The dance can convey the location of nectar, pollen, resin, water, or a new nest site. How other bees interpret these signals varies with the vigor of the dance as well as each bee's current motivational state. Furthermore, the honeybee appears to interpret different destinations as options that can be weighed in terms of evidence bearing on their likelihood or their current value. When a foraging bee that has been trained to visit a feeding station is unexpectedly displaced, it displays search components in the direction of both the hive and the feeder, and ultimately chooses one or the other (Menzel et al. 2005). This behavior can be interpreted as each individual bee differentially investing in each of the possible options, before selecting one and committing to that action. Even more astonishing, bees appear to compute social decisions, in a process akin to voting, to choose a new nest site when swarming (Lindauer 1955).

Presently, we know little about how these processes are encoded in the honeybee brain. The bee brain includes an analog of the vertebrate dopamine 
system, the octopamine system, which apparently serves a similar role in value learning (Menzel and Giurfa 2001). How this valuation system might influence valuation and decision processes carried out elsewhere in the bee brain remains to be studied. One clue to the design of the neural architecture supporting value-based decisions in both vertebrates and invertebrates is that neuromodulatory systems such as dopamine and octopamine project widely and appear to broadcast a message related to values and emotions of a single class (e.g., appetitive, aversive; Schultz and Dickinson 2000). Similar principles characterize the serotonergic, cholinergic, and norepinephrine systems, which appear to promote changes in the value of different options or behavioral strategies. In this scheme, the message computed by any individual neuron may change with respect to the weighting of inputs and channeling of outputs by altering modulatory states. Future progress in understanding the principles governing decision making and how the decision process is embodied in large networks of neurons in humans and other vertebrates will profit from an understanding of how decision making is accomplished by a limited number of highly specialized neurons in the invertebrate nervous system (Menzel and Giurfa 2006). In particular, one may ask whether the computational role of individual neurons embedded in their anatomical and functional network may change with modulatory states and expected outcomes.

\section{Extending Neuronal Models of Decision Making to Legal Issues and Group Processes}

Can an understanding of the neurobiology of decision making have clear implications for decision making at the group or institutional level?

Institutions are humanly devised formal (rules, laws, constitutions) or informal (norms of behavior, conventions, and self imposed codes of conduct) constraints that govern the behavior of two or more individuals (North 1990). These constraints define the incentive structure of societies and, specifically, economies. Institutions are a central concern for law, which serves as the formal regime for political rule making and enforcement. In general, understanding the neurobiology of decision making could be important for understanding how institutions have evolved over time (see McElreath et al., this volume), as well as how institutions impact human decision making (e.g., judge and jury decision making) and behavior (see Lubell et al., this volume). At the present time, it is not possible to state whether neurobiology can ultimately help us reach a better understanding of institutional decision making. However, in the following sections, we explore current neuroscientific data and their potential impacts on institutional decision making. 


\section{Evolution and Formulation of Institutions and Neural Models of Decision Making}

Although institutions may be deliberately and intentionally formulated by people, the evolution and function of institutions might be better understood as emergent phenomena. In other words, institutions evolve and operate in a fashion that cannot be predicted from the decisions made by any single individual. If institutions are truly emergent, this raises the important questions of what we can learn from neurobiological or behavioral studies in animals to explain how institutions have evolved (see McElreath et al., this volume) and whether neurobiology can inform the formulation of rules by political and legal institutions to promote desired social behavior.

These questions have begun to be addressed explicitly in the realm of public policy. As we have discussed in this report, motivation and emotion contribute in important ways to decision making; thus, understanding how the cognitive control of motivation and emotion is implemented in the brain might provide important insights for institutional decision making. If, for example, a society wishes to regulate a particular form of undesirable behavior, knowledge of the neural mechanisms underlying cognitive control may provide important insights in developing legal rules to achieve this goal. Ochsner and Gross (2005), for example, have proposed that cognitive control of emotional states requires both attentional selection of information and cognitive modulation of emotional processes; that is, processes which appear to be implemented by distinct neural systems. To the extent that this model provides an accurate description of the neurobiology of cognitive control, different types of legal rules might be developed to engage these different systems differentially in order to attain desirable goals. In a similar vein, Bernheim and Rangel (2004) recently applied current understanding of the neurobiology of addiction to the formulation of better economic policies on addictive substances. These models suggest that neurobiological understanding, when thoughtfully applied, may positively inform public policy making.

\section{Legal Decision Making and Neural Models of Decision Making}

Understanding the neuronal correlates of decision making may also impact how we address the problem of determining culpability and setting standards of punishment in the legal system. Clearly, people's actions (i.e., the outcome of their decisions) are determined by processes that occur in their brains. This obvious fact raises the troubling question of whether we are responsible for our actions, and it has clear implications for attributing agency and assigning punishment.

The problem can be illustrated by the difference in culpability one would ascribe to a defendant who impulsively acts out his aggression against another person due to a large brain tumor in the medial prefrontal cortex. In this case, 
most people would not hold the defendant responsible for his actions. Contrast this situation with a defendant who commits the same act due to a nonobvious change in neural circuitry as a result of environmental or physiological deficits that arose during development, subliminal brain injury, or genetic factors. Whether this defendant should be treated any differently under the law than the defendant with the obvious brain tumor is a highly contentious and hotly debated issue. Yet, as evidence accumulates favoring the idea that brain states reflect the interaction of genes and environmental influences during development, which means they are thus out of the control of the individual, this legal problem will become thornier indeed. Witness the interaction between the expression of different genetic variants involved in neuromodulatory function (e.g., monoamine oxidase A, serotonin transporter gene), developmental stress, and likelihood of being incarcerated for violence (Caspi et al. 2002).

It is perhaps worth considering the various goals of the legal system in this regard. If the goal is to mitigate the risk of recidivism, then scientific evidence supporting a clear relationship between broken connections among genes, brains, and appropriate action should play an important role in the legal process. If the goal of the legal system is, however, to determine moral agency and thus culpability for antisocial behaviors (perhaps to deter such behaviors in the society) absent of preventing future illegal behavior, then the utility of neuroscientific evidence becomes less clear. Current models of perceptual- and value-based decision making may help guide the assessment of neuroscientific evidence favoring a link between genes, development, brain states, and action. Yet even as neuroscience begins to explain the mental capacities that underlie agency, motivation, and initiative - and hence responsibility and free will - it does not explain away these phenomena. Insights from neurobiology may augment the discussion of such legal concepts as negligence, but it should not override traditional approaches.

\section{Conclusions}

Neurobiologists now understand, in great detail, how simple decisions are made by individual neurons and groups of neurons in several well-defined experimental contexts. This understanding has proceeded from the simultaneous application of formal computational models to both behavior and the activity patterns of individual neurons. This achievement was unthinkable fifty years ago, and thus we should feel optimistic that even more progress will be made over the next half century. Despite this optimism, significant challenges remain. Most importantly, a wide gulf remains between the simple decisions typically studied in the laboratory and the complex decisions made in the real world. Bridging this gap will require concerted integration across multiple disciplines and levels of analysis. This has already begun in the fields of neuroscience, psychology, and economics, but only a brave few have crossed the chasms 
separating these fields from anthropology, evolutionary biology, political science, and the law. Equally important to dispel natural fears of a brave new world of biological determinism and moral ambiguity, our boldness in crossing these boundaries must be bolstered by the quality of our science and the care with which our conclusions are communicated to the wider community.

\section{References}

Amorapanth, P., J. E. LeDoux, and K. Nadar. 2000. Different lateral amygdala outputs mediate reactions and actions elicited by a fear-arousing stimulus. Nature Neurosci. 3(1):74-79.

Bechara, A., and A. R. Damasio. 2005. The Somatic Marker Hypothesis: A Neural Theory of Economic Decision. Games Econ. Behav. 52: 336-372.

Bechara, A., A. R. Damasio, H. Damasio, and S. W. Anderson. 1994. Insensitivity to future consequences following damage to human prefrontal cortex. Cognition 50:7-15.

Bendiksby, M. S. and M. L. Platt 2006. Neural correlates of reward and attention in macaque area LIP. Neuropsychologia 44(12):2411-2420.

Bernheim, B. D., and A. Rangel. 2004. Addiction and cue-triggered decision processes. Am. Econ. Rev. 94(5):1558-1590.

Berridge, K. C., and T. E. Robinson. 2003. Parsing reward. Trends Neurosci. 26(9):407-513.

Busemeyer, J., and J. Townsend. 1995. Decision field theory. In: Mind as Motion, ed. R. Port and T. van Gelder. Cambridge, MA: MIT Press.

Busemeyer, J. R., and J. T. Townsend. 1993. Decision field theory: A dynamic-cognitive approach to decision making in an uncertain environment. Psychol. Rev. 100(3):432-459.

Caspi, A., J. McClay, T. E. Moffitt, et al. 2002. Role of genotype in the cycle of violence in maltreated children. Science 297(5582):851-854.

Cohen, J. D. 2005. The vulcanization of the human brain: A neural perspective on interactions between cognition and emotion. J. Econ. Persp. 4:3-24.

Cosmides, L., and J. Tooby. 2000. Evolutionary psychology and the emotions. In: Handbook of Emotions (2nd ed.), ed. M. Lewis and J. M. Haviland-Jones, pp. 91-115. New York: Guliford.

Cowey, A. and P. Stoerig. 1991. The neurobiology of blindsight. Trends Neurosci. 14(4):140-145.

Damasio, A. R. 1996. The somatic marker hypothesis and the possible functions of the prefrontal cortex. Phil. Trans. Roy. Soc. Lond. B 351(1346):1413-1420.

Daw, N. D., S. Kakde, and P. Dayan. 2002. Opponent interactions between serotonin and dopamine. Neural Networks 15(4-6):603-616.

Dehaene, S. 2007. Symbols and quantities in parietal cortex: Elements of a mathematical theory of number representation and manipulation. In: Attention and Performance XXII. Sensorimotor Foundations of Higher Cognition, ed. P. Haggard and Y. Rossetti. Cambridge, MA.: Harvard Univ. Press, in press

Dehaene, S. and L. Naccache 2001. Towards a cognitive neuroscience of consciousness: Basic evidence and a workspace framework. Cognition 79:1-37.

Dehaene, S., L. Naccache, G. H. Le Clec, et al. 1998. Imaging unconscious semantic priming. Nature 395:597-600. 
Del Cul, A., S. Baillet, and S. Dehaene. 2007. Brain dynamics underlying the non-linear threshold for access to consciousness. PLoS Biol., in press.

Ding, L., and O. Hikosaka. 2006. Comparison of reward modulation in the frontal eye field and caudate of the macaque. J. Neurosci. 26(25):6695-6703.

Fries, P., J. H. Reynolds, A. E. Rorie, and R. Desimone. 2001. Modulation of oscillatory neuronal synchronization by selective visual attention. Science 291:1560-1563.

Fries, P., P. R. Roelfsema, A. K. Engel, P. König, P., and W. Singer. 1997. Synchronization of oscillatory responses in visual cortex correlates with perception in interocular rivalry. Proc. Natl. Acad. Sci. 94:12,699-12,704.

Fries, P., J. H. Schröder, P. R. Roelfsema, W. Singer, and A. K. Engel. 2002. Oscillatory neuronal synchronization in primary visual cortex as a correlate of stimulus selection. J. Neurosci. 22(9):3739-3754.

Gallagher, H. L., and C. D. Frith. 2003. Functional imaging of "theory of mind." Trends Cogn. Sci. 7(2):77-83.

Glimcher, P. W. 2003. The neurobiology of visual saccadic decision making. Ann. Rev. Neurosci. 26:133-179.

Glimcher, P. W. 2005. Indeterminacy in Brain and Behavior. Ann. Rev. Psychol. 56:25-56.

Gold, J. I., and M. N. Shadlen. 2007. The neural basis of decision making. Ann. Rev. Neurosci. 30:535-574.

Heekeren, H. R., S. Marrett, P. A. Bandettini, and L. G. Ungerleider. 2004. A general mechanism for perceptual decision making in the human brain. Nature 431:859-862.

Horwitz, G. D., and W. T. Newsome. 2001. Target selection for saccadic eye movements: Prelude activity in the superior colliculus during a direction-discrimination task. J. Neurophysiol. 86:2543-2558.

Huettel, S. A., C. J. Stowe, E. M. Gordon, B. T. Warner, and M. L. Platt. 2006. Neural signatures of economic preferences for risk and ambiguity. Neuron 49:765-775.

Huk, A. C., and M. N. Shadlen. 2005. Neural activity in macaque parietal cortex reflects temporal integration of visual motion signals during perceptual decision making. $J$. Neurosci. 25:10,420-10,436.

Kim, J.-N., and M. N. Shadlen. 1999. Neural correlates of a decision in the dorsolateral prefrontal cortex of the macaque. Nature Neurosci. 2(2):176-185.

Kouider, S., and S. Dehaene. 2007. Levels of processing during non-conscious perception: A critical review of visual masking. Phil. Trans. R. Soc. Lond. B 362(1481):857-875.

Kunde, W. 2003. Sequential modulations of stimulus-response correspondence effects depend on awareness of response conflict. Psychon. Bull. Rev. 10(1):198-205.

Lau, H. C., and R. E. Passingham. 2007. Unconscious activation of the cognitive control system in the human prefrontal cortex. J. Neurosci. 27(21):5805-5811.

LeDoux, J. E. 1996. The Emotional Brain. New York: Simon and Schuster.

Lerner, J.S., and D. Keltner. 2001. Fear, anger, and risk. J. Pers. Soc. Psychol. 81:146-159.

Lowenstein, G. A., and J. S. Lerner. 2003. The role of affect in decision making. In: Handbook of Affective Sciences, ed. R. J. Davidson, K. R. Scherer, and H. H. Goldsmith. Oxford: Oxford Univ. Press.

Lindauer, M. 1955. Schwarmbienen auf Wohnungssuche. Physiol. 37:263-324.

Logothetis, N. K., and J. D. Schall. 1989. Neuronal correlates of subjective visual perception. Science 45(4919): 761-763. 
Luce, R. D. 1959. Individual Choice Behavior: A Theoretical Analysis. New York: Wiley.

Luce, R. D., and P. Suppes. 1965. Preference, utility, and subjective probability. In: Handbook of Mathematical Psychology, vol. 3. ed. R. Luce, R. Bush, and E. Galanter, New York: Wiley.

Machens, C. K., R. Romo, and C. D. Brody. 2005. Flexible control of mutual inhibition: A neural model of two-interval discrimination. Science 307(5712):1121-1124.

Manski, C. 1977. The Structure of Random Utility Models. Theory and Decision 8:229-254.

Maynard Smith, J. 1982. Evolution and the Theory of Games. Cambridge: Cambridge Univ. Press.

Mazurek M. E., J. D. Roitman, J. Ditterich, and M. N. Shadlen. 2003. A role for neural integrators in perceptual decision making. Cerebral Cortex 13:1257-1269.

McCabe, K., and V. L. Smith. 2000. A two person trust game played by naïve and sophisticated subjects. Proc. Natl. Acad. Sci. (97)7:3777-3781.

McCabe, K., D. Houser, L. Ryan, V. Smith, and T. Trouard. 2001. A functional imaging study of cooperation in two-person reciprocal exchange. Proc. Natl. Acad. Sci. 98:11,832-11,835.

Melloni, L., C. Molina, M. Pena, D. Torres, W. Singer, and E. Rodriguez. 2007. Synchronization of neural activity across cortical areas correlates with conscious perception. J. Neurosci. 27(11):2858-2865.

Menzel, R., B. Brembs, and M. Giurfa. 2007. Cognition in invertebrates. In: Evolution of Nervous Systems, vol. II: Evolution of Nervous Systems in Invertebrates, ed. J. H.Kaas, chapter 1.26, pp. 403-422. Oxford: Academic Press.

Menzel, R., and Giurfa, M. 2001. Cognitive architecture of a mini-brain: The honeybee. Trends Cogn. Sci. 5:62-71.

Menzel, R., and Giurfa, M. 2006. Dimensions of cognition in an insect, the honeybee. Behav. Cogn. Neurosci. Rev. 5:24-40.

Menzel, R., U. Greggers, A. Smith, S. Berger, R. Brandt et al. 2005. Honey bees navigate according to a map-like spatial memory. Proc. Natl. Acad. Sci. 102(8):3040-3045.

Merikle, P. M., and S. Joordens. 1997. Parallels between perception without attention and perception without awareness. Conscious Cogn. 6(2-3):219-236.

Niv, Y., N. D. Daw, D. Joel, and P. Dayan. 2006. Tonic dopamine: Opportunity costs and the control of response vigor. Psychopharm. 191(3):507-520.

North, D. C. 1990. A Transaction cost theory of politics. Washington, St. Louis: School of Business and Political Economy, Papers 144.

Ochsner, K. N., and J. J. Gross. 2005. The cognitive control of emotion. Trends Cogn. Sci. 9(5):242-249.

Parker, A. J., and W. T. Newsome. 1998. Sense and the single neuron: Probing the physiology of perception. Ann. Rev. Neurosci. 21:227-277.

Pessiglione, M., L. Schmidt, B. Draganski, et al. 2007. How the brain translates money into force: A neuroimaging study of subliminal motivation. Science 316(5826):904-906.

Platt, M. L., and P. W. Glimcher. 1999. Neural correlates of decision variables in parietal cortex. Nature 400(6741):233-238.

Ratcliff, R. 1978. A theory of memory retrieval. Psychol. Rev. 85:59-108.

Ratcliff, R., A. Cherian, and M. Segraves. 2003. A comparison of macaque behavior and superior colliculus neuronal activity to predictions from models of two-choice decisions. J. Neurophysiol. 90(3):1392-1407. 
Ratcliff, R., A. Thapar, and G. McKoon. 2006. Aging and individual differences in rapid two-choice decisions. Psychon. Bull. Rev. 13(4):626-635.

Roesch, M. R., and C. R. Olson. 2003. Impact of expected reward on neuronal activity in prefrontal cortex, frontal and supplementary eye fields and premotor cortex. $J$. Neurophysiol. 90(3):1766-1789.

Roitman, J. D., and M. N. Shadlen. 2002. Response of neurons in the lateral intraparietal area during a combined visual discrimination reaction time task. J. Neurosci. 22(21):9475-9489.

Romo, R., A. Hernández, and A. Zainos. 2004. Neuronal correlates of a perceptual decision in ventral premotor cortex. Neuron 41(1):165-173.

Romo, R., and E. Salinas. 2003. Flutter discrimination: Neural codes, perception, memory and decision making. Nature Rev. Neurosci. 4:203-218.

Scherer, K. R. 2000. Psychological models of emotion. In: The Neuropsychology of Emotion, ed. J. C. Borod, pp. 137-163. New York: Oxford Univ. Press.

Schultz, W., and A. Dickinson. 2000. Neuronal coding of prediction errors. Ann. Rev. Neurosci. 23:473-500.

Sergent, C., S. Baillet, and S. Dehaene. 2005. Timing of the brain events underlying access to consciousness during the attentional blink. Nature Neurosci. 8(10):1391-1400.

Shadlen, M. N., and R. Kiani. 2007. Neurology: An awakening. Nature 448:539-540.

Shadlen, M. N., and W. T. Newsome. 1994. Noise, neural codes and cortical organization. Curr. Opin. Neurobiol. 4:569-579.

Shadlen, M. N., and W. T. Newsome. 1998. The variable discharge of cortical neurons: Implications for connectivity, computation and information coding. J. Neurosci. 18:3870-3896.

Shadlen, M. N., and W. T. Newsome. 2001. Neural basis of a perceptual decision in the parietal cortex (area LIP) of the rhesus monkey. J. Neurophysiol. 86:1916-1936.

Siegler, R. S. 1988. Strategy choice procedures and the development of multiplication skill. J. Exp. Psychol.: Gen. 117(3):258-275.

Sigman, M., and S. Dehaene. 2005. Parsing a cognitive task: A characterization of the mind's bottleneck. PLoS Biol 3(2):e37 doi:10.1371.

Sigman, M., and S. Dehaene. 2006. Dynamics of the central bottleneck: Dual-task and task uncertainty. PLoS Biol 4(7):e220 doi:10.1371.

Singer, W. 2007. Large-scale temporal coordination of cortical activity as a prerequisite for conscious experience. In: The Blackwell Companion to Consciousness, ed. M. Velmans and S. Schneider, pp. 605-615. Oxford: Blackwell.

Smith, P. L., and R. Ratcliff. 2004. Psychology and neurobiology of simple decisions. Trends Neurosci. 27:161-168.

Sugrue, L. P., G. S. Corrado, and W. T. Newsome. 2004. Matching behavior and the representation of value in the parietal cortex. Science 304(5678): 1782-1787.

Tononi, G., R. Srinivasan, D. P. Russell, and G. M. Edelman. 1998. Investigating neural correlates of conscious perception by frequency-tagged neuromagnetic responses. Proc. Natl. Acad. Sci. 95:3198-3203.

Tomb, I., M. Hauser, P. Deldin, and A. Caramazza. 2002. Do somatic markers mediate decisions on the gambling task? Nature Neurosci. 5(11):1103-1104.

Vorberg, D., U. Mattler, A. Heinecke, et al. 2003. Different time courses for visual perception and action priming. Proc. Natl. Acad. Sci. 100(10):6275-6280.

Wang, X. J. 2002. Probabilistic decision making by slow reverberation in cortical circuits. Neuron 36(5):955-968.

Wong, K. F., and X. J. Wang. 2006. A recurrent network mechanism of time integration in perceptual decisions. J. Neurosci. 26(4):1314-1328. 